Flow Turbulence Combust

DOI 10.1007/s10494-016-9729-2

published online: 13 April 2016

\title{
Wind Impact on Single Vortices and Counterrotating Vortex Pairs in Ground Proximity
}

\author{
Frank Holzäpfel $^{1}$, Nikola Tchipev ${ }^{2}$, Anton Stephan ${ }^{3}$
}

\begin{abstract}
Wall-resolved large eddy simulations are employed to investigate the behaviour of wake vortices and single vortices in ground proximity at a variety of wind conditions. The six considered strengths of wind, ranging between 0.5 and 4 times the initial wake vortex descent speed, $w_{0}$, include practically and theoretically significant wind speeds. A crosswind of $0.5 w_{0}$ may lead to windward stall posing a potential hazard to subsequently landing aircraft, whereas theoretical considerations predict that at $4 w_{0}$ the rebound of the luff vortex is completely suppressed. The same range of wind speeds is also used to investigate the effects of headwind and diagonal wind in order to discriminate between effects of environmental turbulence increasing with wind speed and the direction of the wind shear. The study has been complemented by a number of single vortex computations in order to differentiate between effects related to the mutual interaction of the vortex pair and the individual vortices with the turbulent boundary layer flow. It is shown that vortex ascent, descent, rebound and decay characteristics are controlled by (i) the interaction of the vortices with secondary vorticity detaching from the ground, (ii) the redistribution of vorticity of the boundary layer which is altering the path of the primary vortices by mutual velocity induction, and (iii) the interaction of the vortices with the environmental turbulence.
\end{abstract}

Keywords wake vortices, large eddy simulation, headwind, crosswind, ground effect

\section{Introduction}

Aircraft trailing vortices, generated as an unavoidable consequence of lift, pose a potential risk to following aircraft. The current separation standards between consecutive aircraft are still being heavily influenced by the early studies carried out in the 1970s, with additional refinements introduced in the 1980s and 1990s [1]. These aircraft separations limit the capacity of congested airports in a rapidly growing aeronautical environment. The most likely economic scenario for the future European airport demand [2] indicates that there will be around 1.9 million unaccommodated flights in 2035, constituting approximately $12 \%$ of the expected demand.

The highest risk to encounter wake vortices prevails in ground proximity, where the vortices cannot descend below the glide path but tend to rebound due to the interaction with the ground surface. Weak crosswinds may compensate the self-induced lateral propagation of the upwind vortex, such that it may hover over the runway directly in the flight path of the following aircraft $[3,4]$. As a consequence, the incident reporting scheme established by NATS (National Air Traffic Service) reports most encounters at flight altitudes below $300 \mathrm{ft}[5,6]$ with a particular clustering even below $100 \mathrm{ft}$ [7].

A few years ago ICAO (International Civil Aviation Organisation) has initiated a process for the harmonization of wake turbulence separations termed RECAT (Re-Categorization). RECAT phase 1 refers to the classification into

\footnotetext{
${ }^{1}$ Institut für Physik der Atmosphäre, Deutsches Zentrum für Luft- und Raumfahrt, 82234 Oberpfaffenhofen, Germany, frank.holzaepfel@dlr.de, Tel. +49 8153 28-2529, Fax +49 8153 28-1841

${ }^{2}$ Institut für Physik der Atmosphäre, Deutsches Zentrum für Luft- und Raumfahrt, 82234 Oberpfaffenhofen, Germany, currently: Technische Universität München, Department of Informatics, 85748 Garching, Germany

${ }^{3}$ Institut für Physik der Atmosphäre, Deutsches Zentrum für Luft- und Raumfahrt, 82234 Oberpfaffenhofen, Germany
} 
six categories considering besides the weight the approach speed, wing characteristics and in parts also the rolling moment exerted on following aircraft. RECAT 1 has been implemented at the US airports Memphis, Louisville, Cincinnati, Atlanta, Houston, New York Metroplex, and Charlotte followed by others [8]. The European wake recategorization, RECAT-EU, which shall increase runway capacity by $3 \%$ to $8 \%$ and better protect small aircraft, shall be introduced in December 2015 at Charles de Gaules airport [9]. The developments of RECAT I, RECAT II (static pair-wise separations), Time-Based Separations (TBS) as well as the wake vortex investigations and safety cases for the A380 and the B747-8 all focus on wake vortex evolution in ground proximity as the most relevant phase of flight with respect to wake vortex risks.

The long-term goal of the initiative RECAT III plans for dynamic pair-wise separations that consider dynamically the aircraft type pairing and the prevailing environmental conditions. For an optimum exploitation of the dynamic pair-wise separations concept, full understanding of wake vortex behaviour during final approach and touchdown is mandatory. An analysis of DLR's (Deutsches Zentrum für Luft- und Raumfahrt) wake vortex advisory system WSVBS (Wirbelschleppen-Vorhersage und Beobachtungs-System) demonstrates that in 57\% - 70\% of landings wake vortex behaviour in ground proximity impedes reduced separations [10]. So there is substantial impetus for thorough investigations of wake vortex behaviour in ground proximity from a flight safety perspective and the optimization of wake vortex separations.

But these types of flows are also of fundamental interest to fluid dynamicists as they constitute relatively simple configurations of coherent vortex structures being embedded in incoherent, i.e., turbulent environments. The study of such flows yields improved understanding of elementary vortex dynamics that is also relevant in more complex transitional and turbulent flows. However, already simple vortices introduced in proximity to a wall feature intriguing three-dimensional developments. They induce a vorticity layer at the surface which first causes the vortices to move along the surface driven by mutual velocity induction. Single vortices are transported parallel along the surface in the direction of the flow they induce above the surface. Counterrotating vortex pairs diverge during their approach to the ground following the hyperbolic trajectory of classical inviscid theory. Because of an adverse pressure gradient, the vorticity layer may separate from the surface leading to the formation of secondary vortices $[11,12]$. The detached secondary vortices may orbit around the primary vortices and the newly formed unequal vortex pairs rebound in a spiraling motion.

The presence of an ambient crosswind induces another boundary layer corresponding to a vorticity layer at the ground. The sudden eruption of vortex-induced wall vorticity is faster and more intense for vortices with opposite signed vorticity relative to the crosswind shear-generated vorticity and in turn attenuated for vortices with equally signed vorticity. The reason for this asymmetry is that crosswind shear supports the formation of secondary vorticity at the wall for the former vortex and weakens it for the latter one. On top of this, the primary vortices may redistribute the crosswind vorticity which in turn alters the trajectories of the vortices [13]. This effect is mostly relevant for cases with strong crosswinds and for vortices that do not generate detaching secondary vortices.

During approach and landing the situation is even more complicated because of the inclination of the wake vortices and the end effects occurring once the aircraft touches the runway. These end effects trigger instabilities that quickly propagate against flight direction and weaken the vortices [14]. Moreover, the vortices undergo complex deformations and may link with the ground. All these phenomena are not within the scope of this more fundamental investigation.

The first investigations on ground effect have been performed by Prandtl [15] and Wieselsberger [16] employing a modification of Prandtl's lifting line theory. The first flight experiments [17] and laboratory observations [18, 19] date about 50 years later. These early works were followed by numerical simulations [12, 14, 20-31], laboratory experiments [22, 32], and field experiments [3, 4, 33-36]. A survey on vortex interactions with walls is given in [11]. Two dimensional simulations [13,21-25] already point out asymmetric wake vortex behaviour caused by crosswind shear. Three-dimensional simulations illustrate that secondary vortices are susceptible to spatial crosswind variations [26] or short-wavelength instabilities [27] which both deform the secondary vortices and subsequently lead to reconnection of primary and secondary vortices. Linking of vortices with the ground is reported at moderate to high ambient turbulence levels [14, 28]. Wall-resolved large eddy simulations (LES) considering effects of headwind and crosswind are reported in [31]. Further, it is described that vortices decay more rapidly near the ground and that the decay rate only weakly depends on ambient turbulence [3, 28, 34, 35]. [12] demonstrates that so-called streaks, developing in the atmospheric boundary layer close to the ground, trigger the transformation of the longitudinal secondary vortices into $\Omega$-shaped vortices that actively approach and wrap around the primary wake vortices and thus accelerate wake vortex decay. Hybrid simulations of wake-vortex evolution during approach and landing reveal the relevance of end-effects triggered during touchdown for wake vortex decay in ground proximity [14].

The current study employs wall-resolved LES in order to investigate wake vortex behaviour in ground proximity at a variety of wind conditions. Wall-resolved LES employ DNS (direct numerical simulation) like grid refinement 
at the ground and thus enable the resolution of near-wall streaks that have proven to play a substantial role for vortex decay in ground proximity [12]. The downside of wall-resolved LES is that the viscosity has to be adjusted artificially high leading to relatively low Reynolds numbers. Section 2 introduces the applied LES method and the selected initial conditions. Section 3 discusses headwind effects on single vortices and vortex pairs where mainly the headwind turbulence modifies vortex behaviour compared to the relatively simple vortex dynamics in a quiescent environment. The introduction of crosswind shear vorticity in section 4 adds complexity as it modifies the strength of the generated secondary vortices and directly alters the vortex trajectories by redistribution processes. Finally, section 5 investigates how the effects of headwind and crosswind add up in a diagonal wind situation and how this can be modelled by simple means. A precursor version of this article has been presented at the 7th AIAA Atmospheric and Space Environments Conference [37]. More details of this study can be found in [38].

\section{Numerical Method and Initial Conditions}

\subsection{Numerical Method}

For this study large eddy simulation (LES) is employed because it describes the interaction of rotation and turbulence at least on the resolved scales correctly. Unsteady Reynolds-Averaged-Navier-Stokes simulations (URANS) usually are too dissipative to preserve tight vortex cores for longer times. LES is performed using the incompressible code MGLET [39] which solves the Navier-Stokes equations

$$
\begin{gathered}
\frac{\partial u_{i}}{\partial t}+\frac{\partial\left(u_{i} u_{j}\right)}{\partial x_{j}}=-\frac{1}{\rho_{0}} \frac{\partial p^{\prime}}{\partial x_{i}}+\frac{\partial}{\partial x_{j}}\left[\left(v+v_{t}\right) 2 S_{i j}\right] \\
\frac{\partial u_{j}}{\partial x_{j}}=0
\end{gathered}
$$

where $u_{i}$ and $p$ ' represent the velocity components in three spatial directions ( $i=1,2$ or 3$)$ and pressure, respectively. The prime for pressure indicates that it is defined by the deviation from the reference state, hence $p=p_{0}{ }^{+} p^{\prime}$. Kinematic viscosity in the momentum equations is defined by the sum of molecular viscosity and eddy viscosity obtained from a subgrid-scale model. $S_{i j}=\left(\partial u_{i} / \partial x_{j}+\partial u_{j} / \partial x_{i}\right) / 2$ denotes the strain rate tensor.

The above equations are solved by a finite-volume approach with a fourth-order finite-volume compact scheme [40]. A split-interface algorithm is used for the parallelization of a tri-diagonal system for the compact scheme, which realizes good overhead time and scalability in parallel environments. In addition, a divergence free interpolation is employed for obtaining advection velocity, which ensures conservation of velocity and passive tracer fields. The pressure field is obtained by the velocity-pressure iteration method [41]. For time integration the thirdorder Runge-Kutta method is used [42]. The Lagrangian dynamic model [43] is employed as turbulence closure which prevents excessive eddy viscosity in the vortex cores. The closure accumulates the required averages of subgrid model coefficients along flow pathlines. This enables the Lagrangian dynamic model to distinguish the centrifugally stable vortex core regions as well as boundary layer flows from the external turbulent flow. All computations are performed in parallel by a domain decomposition approach.

\subsection{Computational Domain and Boundary Conditions}

The computational domain displayed in Fig. 1 consists of a box with the dimensions $\left(L_{x}, L_{y}, L_{z}\right)=\left(8 b_{0}, 8 b_{0}, 3 b_{0}\right)=$ ( $384 \mathrm{~m}, 384 \mathrm{~m}, 144 \mathrm{~m}$ ), where $b_{0}=48 \mathrm{~m}$ corresponds to the initial vortex separation. For strong crosswinds of 2 initial vortex descent speeds $w_{0}=1.5 \mathrm{~m} / \mathrm{s}$ and more the lee vortex may catch up the luff vortex when advected across the periodic boundary. Therefore in these cases the lateral dimensions of the domain were doubled. The boundary conditions represent the typical "half-channel flow" scenario with a no-slip condition at the ground, free-slip condition at the top and periodic conditions along all horizontal boundaries. 


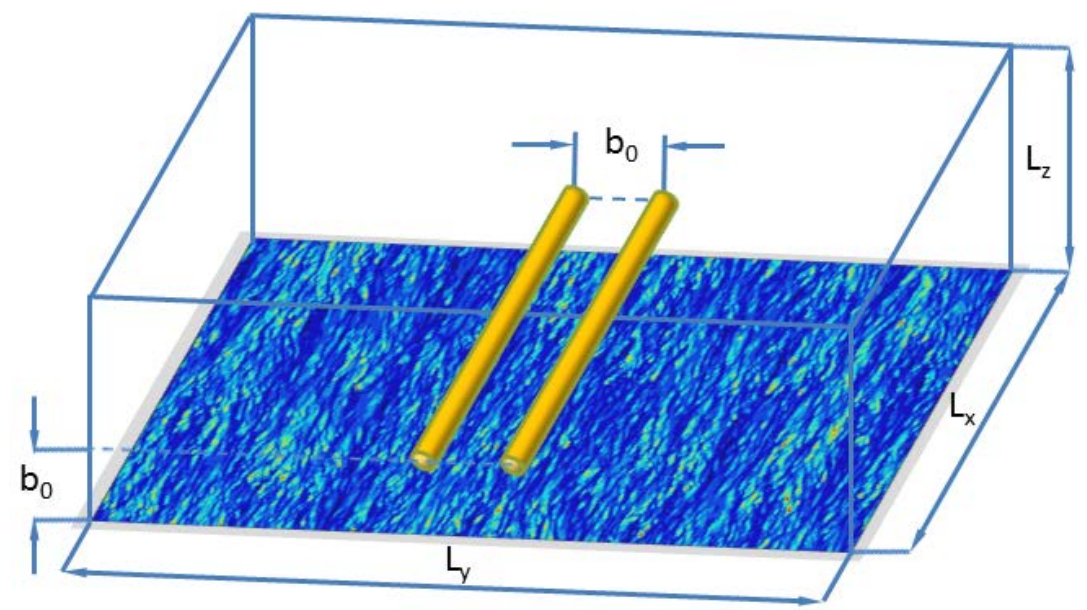

Fig. 1 Computational domain and initial vortex position with headwind streaks at the ground

The employed grid spacing is constant across all investigated scenarios. In the longitudinal and lateral directions it is set to $1 / 64 b_{0}(0.75 \mathrm{~m})$. In the vertical direction it is equal to $1 / 64 b_{0}$ above an altitude of $b_{0}$, and refined towards the ground in a geometric fashion down to $1 / 320 b_{0}(0.15 \mathrm{~m})$. This is needed to satisfy the typical requirement $z_{1}^{+} \approx 1$ of wall-resolved LES. Measurements with five anemometers mounted at different altitudes above ground indicate that the wake vortex boundary layer is much thinner than that of the ambient wind [35].

\subsection{Turbulent Boundary Layer}

Before the vortices are inserted the environmental wind is simulated as a turbulent half channel flow driven by an imposed pressure gradient. The presimulation is started from a slightly perturbed logarithmic wind profile and continued until the near-wall streaks (see Fig. 1) have appeared and turbulent eddies are present in the entire flow. These near-wall streaks have proven to play a substantial role for vortex decay in ground proximity [12] because they trigger the formation of $\Omega$-shaped secondary vortices that wrap around the primary vortices and weaken them by turbulent interaction. Good agreement of simulations with the logarithmic layer and the viscous sublayer is achieved. The wind profiles resulting from the initialised low Reynolds number half channel flow (see section 2.4) compare with real-life log wind profiles with a roughness length of about $0.01 \mathrm{~m}$, constituting a typical value of an airport.

The investigated wind speeds of headwind and crosswind amount to $0.5,1, \sqrt{2}, 2,3$, and 4 times the initial vortex descent speed $w_{0}$ at a height of one vortex separation $b_{0}$ above ground. At the standard height of operational wind measurements of $10 \mathrm{~m}$ the wind speed amounts to about $80 \%$ of the wind speed prevailing at the height of $b_{0}$. Diagonal wind is considered at the speeds of $1, \sqrt{2}$, and 2 times $w_{0}$. In the legends of the plots $C$ denotes crosswind, $\mathrm{H}$ headwind, D diagonal wind, and $\mathrm{N}$ no wind, where e.g. C05 denotes a crosswind with a wind speed of $0.5 w_{0}$. Case $\mathrm{N}$ without wind lacks any turbulence and as such represents an academic reference case that would not occur in reality because there always turbulence of the aircraft flow and the engine jets will be entrained into the vortices and at least a minimum of environmental turbulence will prevail. However, case $\mathrm{N}$ provides added value by giving insights into the asymptotical behaviour of the vortices not impacted by turbulent disturbances and thus reveals the role of turbulence for wake vortex behaviour in ground proximity in a way only accessible by numerical simulations. Further, case N provides a reference connecting previous 2D or laminar simulations [11, 13, 19-24, 29, 30 ] and current $3 \mathrm{D}$ turbulent investigations. Within the considered wind speed range the friction velocity $\mathrm{u}_{\tau}$ varies between $0.04 \mathrm{~m} / \mathrm{s}$ to $0.29 \mathrm{~m} / \mathrm{s}$ and the turbulence kinetic energy between $0.002 \mathrm{~m}^{2} / \mathrm{s}^{2}$ to $0.035 \mathrm{~m}^{2} / \mathrm{s}^{2}$.

\subsection{Vortex Initialization}

The vortices are initialized by superimposing Lamb-Oseen vortices with a circulation of about $\Gamma_{0}=460 \mathrm{~m}^{2} / \mathrm{s}$ and a vortex core radius of $r_{c}=3.0 \mathrm{~m}$ to the turbulent boundary layer flow. Wake vortices are modeled by superposition of a pair of counterrotating Lamb-Oseen vortices with a vortex separation $b_{0}=48 \mathrm{~m}$. All vortices are initialized at a 
height of one vortex separation above ground (see Fig. 1). With the selected wake vortex parameters corresponding aircraft would fall into the ICAO weight category heavy.

Several tangential velocity profiles have been suggested to initialize wake vortices and there is no general consensus on the "right" vortex model [44]. Numerical simulations of wake vortex roll-up [45] demonstrate that the evolving tangential velocity profiles are fitted well by a sequence of different analytical vortex models depending on the vortex age (Jacquin's multiple-scale vortex model, Lamb-Oseen vortex, Hallock-Burnham vortex) with an excellent agreement of Lamb-Oseen vortices at a normalized vortex age of $t^{*}=0.4$ (normalization see below). This suggests that during vortex formation the tangential velocity profiles may undergo an evolution across different vortex models. The selected vortex core size of $r_{c} / b_{0}=0.0625$ satisfies the equilibrium of the energy consumed by the induced drag of a slightly outboard loaded wing with a spanwise load factor of $s=0.84$ and the cross-flow kinetic energy of the vortices [46]. On the other hand, vortex core size measurements suggest that the cores may be as compact as one percent of the wingspan of the aircraft [47].

The Reynolds number is limited by the resolution requirements of the wall-resolved LES and set to $R e_{\Gamma}=\Gamma_{0} / v=$ 20.000 with a kinematic viscosity of $v=0.023 \mathrm{~m}^{2} / \mathrm{s}$. If the grid resolution does not match the Reynolds number, a major component of vortex evolution in ground proximity, namely the evolution of the secondary vorticity, is not sufficiently resolved and gets lost by numerical dissipation after the detachment of the secondary vortex from the ground.

For generality wake vortex predictions are formulated in normalized form denoted by an *, where the characteristic scales are based on initial vortex separation, $b_{0}$, and circulation, $\Gamma_{0}$, leading to the timescale $t_{0}=2 \pi b_{0}{ }^{2} /$ $\Gamma_{0}=31 \mathrm{~s}$. The velocity scale is based on the initial descent speed of the vortex pair $w_{0}=\Gamma_{0} / 2 \pi b_{0}=1.5 \mathrm{~m} / \mathrm{s}$. Circulation is averaged over circles with radii from 5 to $15 \mathrm{~m}$. Benefits and drawbacks of radii-averaged circulation definitions are discussed in [48].

Describing the vortex-wind-interaction as a superposition of vortex flow and half-channel flow, one has to specify the wind strength given a vortex Reynolds number. This is done by choosing $\left(\mathrm{b}_{0}, \delta, \Gamma_{0}, v\right)$ and hence $\mathrm{w}_{0}=$ $\Gamma_{0} / 2 \pi b_{0}$. The Reynolds number of the wind is then given by $\operatorname{Re}_{\tau}=u_{\tau} \delta / v$ ( $\delta$ equals the half channel width), which in turn is determined by the logarithmic wind profile by defining the wind speed $\mathrm{u}=\mathrm{w}_{0}$ at $\mathrm{z}=\mathrm{b}_{0}$

$$
\frac{u}{u_{\tau}}=\frac{1}{0.4} \log \left(\frac{z u_{\tau}}{v}\right)+5.5
$$

From this formula we see that the choice of $\mathrm{b}_{0}, \delta$, and $\Gamma_{0}\left(\right.$ via $\left.\mathrm{w}_{0}\right)$ together with $v$ determines the wind profile. Also the relative position of the vortices in the wind profile is determined. Hence the entire system is characterized by the tuple $\left(b_{0}, \delta, \Gamma_{0}, v\right)$ or $\left(\operatorname{Re}_{\tau}, \operatorname{Re}_{\Gamma}\right)$. To link this to a real situation it is convenient to use $b_{0}$ and $\Gamma_{0}\left(\delta / b_{0}\right.$ simply sets an appropriate distance between the vortices and the upper domain boundary). For us this gives a better basis for interpretation of the flow than starting with abstract numbers like $\left(\operatorname{Re}_{\tau}, \operatorname{Re}_{\Gamma}\right)$. For the sake of clearness we present the simulated initial values we are using for our setup.

For prescribing the initial vortex velocity field, six image vortex pairs in the spanwise direction and two mirror vortices in the direction perpendicular to the ground are taken into account. Vortex core locations are tracked by evaluation of the product of axial vorticity and pressure $\omega_{x} p^{3}$, where prior to the search the pressure was shifted to be entirely negative. Methods to exclude flawed tracking of secondary vortices and the shear layer at the ground are detailed in [38].

\section{Headwind Effects on Single Vortices and Vortex Pairs}

This section considers the interaction of single vortices and counterrotating vortex pairs with the ground in headwind situations. While headwind does not contribute directly to the lateral transport of the vortices it adds the environmental turbulence that has a distinct effect on both vortex transport and decay. In real environments there is always at least some residual turbulence, which may have been generated by very different mechanisms, e.g. convectively driven turbulence or mechanical turbulence induced by enhanced surface roughness introduced by buildings and other obstacles. In crosswind situations the interaction of individual vortices with the crosswind vorticity at the ground makes the vortex evolution asymmetrical and is further complicated by the redistribution of the boundary layer vorticity by the coherent vortices. These effects are discussed in detail in the subsequent section.

Vortices in proximity to a solid surface induce a boundary layer (vorticity layer) at the surface which causes the vortices to move along the surface driven by mutual velocity induction. Single vortices are transported in the direction of the flow they induce above the surface. Consequentially, counterrotating vortex pairs diverge during their approach to the ground following the hyperbolic trajectory of classical inviscid theory. These effects are modeled by introducing image vortices below the surface with opposite rotational directions. Because of an adverse pressure gradient the vorticity layer may separate from the surface leading to the formation of secondary vortices. 
The detached secondary vortices may orbit around the primary vortices and the newly formed unequal vortex pairs rebound.
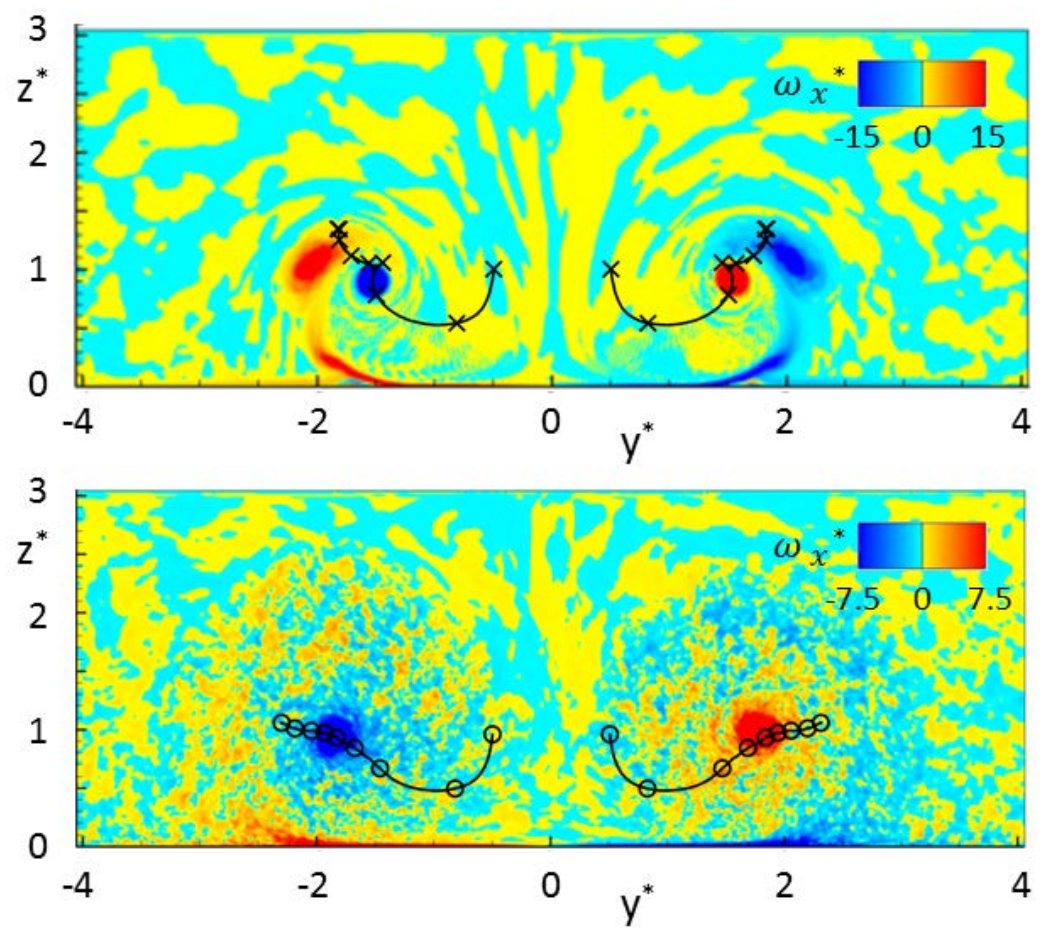

Fig. 2 Vorticity distribution averaged in flight direction for headwind $0.5 \mathrm{w}_{0}$ at $\mathrm{t}^{*}=2.2$ above and for headwind $2 \mathrm{w}_{0}$ at $\mathrm{t}^{*}=3.8$ below complemented by vortex trajectories

Fig. 2 shows snapshots of the behaviour of vortex pairs at headwind strengths of $0.5 w_{0}$ (above) and of $2 w_{0}$ (below) at two different instants of time in terms of axial vorticity that has been averaged along the vortex axes. The figures are complemented by the vortex trajectories denoted by black lines where the symbols are separated by one vortex time scale $t_{0}$. Because the lower headwind in Fig. 2 above generates less environmental turbulence, not only the secondary vortices are still clearly visible outboard but also another set of distinct secondary vortices is about to detach from the ground, the so-called tertiary vortices. While the tertiary vortices orbit around the primary vortices, they induce a second arc of rebound leading to a maximum height of $1.4 b_{0}$.

Due to instabilities triggered by the environmental turbulence, the secondary vortices are progressively deformed and wrapped around the primary vortices losing their coherence as depicted in Fig. 2 below. A detailed discussion of these instabilities is beyond the scope of this paper and can be found in [12]. The enhanced turbulence triggered by a headwind of $2 w_{0}$ disperses the coherence of the secondary and tertiary vortices quickly such that the detachment of secondary vorticity from the ground becomes a more continuous process and the two distinct rebound arcs merge to a continuous diverging rebound motion. A sharp increase of turbulence at the edges of the vortices featuring a patchy structure was also noted within anemometer measurements at John F. Kennedy International Airport [35].

Fig. 3 left depicts the trajectories of the starboard vortex in quiescent air and at 6 different headwind strengths where the symbols are separated by one vortex time scale $t_{0}$. In a quiescent non-turbulent environment the secondary vortices accomplish a substantial fraction of a full orbit around the primary vortices before they lose coherence by stretching and subsequent merger with the primary vortices. As a result the primary vortex executes a full orbit as well. The trajectories of the cases $\mathrm{N}$ and $\mathrm{H} 05$ match almost exactly until about $t^{*}=2.3$, when the rapid circulation decay sets in for the weak headwind case (Fig. 3, right). During the rapid decay phase the coherence of the secondary vortices is getting lost; the secondary vortices wrap around and merge with the primary vortices [12]. As a consequence, the H05 vortices do not descend again. They conduct a second semi-loop driven by the orbiting tertiary vortices. Due to the enhanced altitude of the primary vortices, the orbit of the tertiary pair is much larger and, hence, slower. Ultimately, this second semi-loop takes the primary vortices visibly higher than in the case without wind, N. 

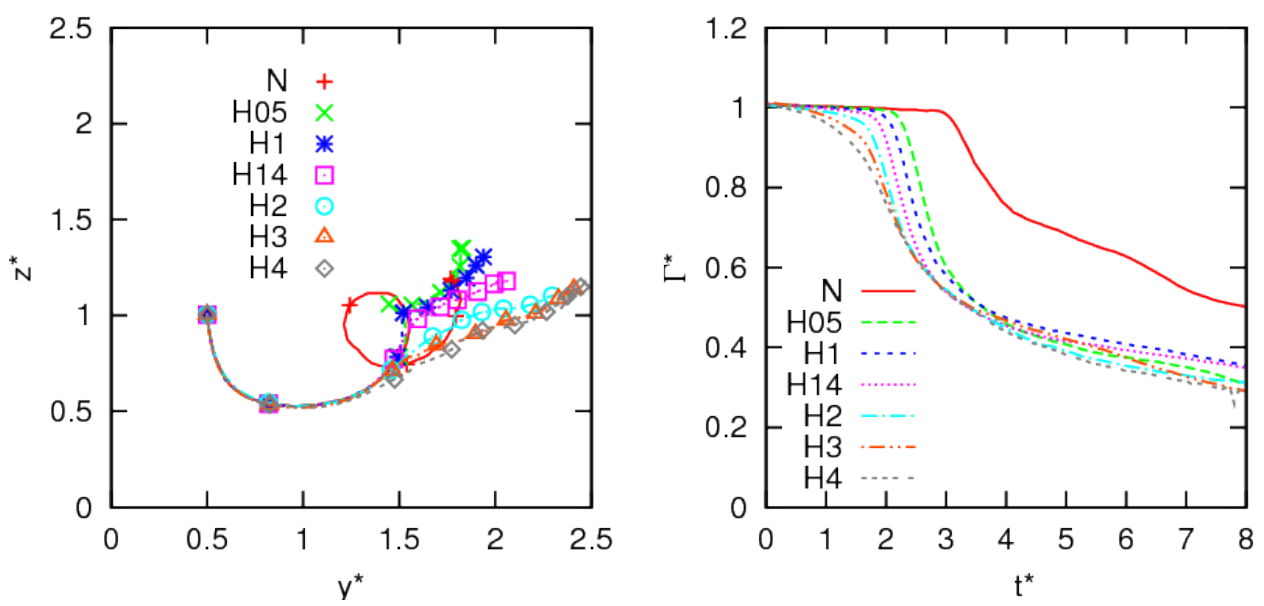

Fig. 3 Wake vortex trajectories and circulation decay for different headwind strengths

With increasing headwind strength the spiraling vortex trajectory becomes less pronounced developing towards a continuous oblique rebound, finally leading to wider divergence but less rebound height. This tendency is explained by the fact that in a quiescent environment the secondary vorticity detaches from the ground as a distinct secondary vortex whereas with increasing headwind/turbulence the detachment progressively develops towards a continuous process, as it is shown in Fig. 2 below and observed in field measurement data [3, 35].

Fig. 3 right indicates that already the weak turbulence generated by a headwind of only $0.5 w_{0}$ accelerates vortex decay substantially compared to a quiescent non-turbulent environment. Note that the slow decay characteristics of case $\mathrm{N}$ in Figs. 3, 4, and 6 cannot be observed in reality and must be considered with care. Even in a quiescent environment the turbulence of the aircraft flow and the engine jets is entrained into the vortices and accelerates vortex decay. With increasing headwind strength the rapid vortex decay, triggered by the annihilation of the wake vortex circulation by secondary vorticity detaching from the ground, sets in earlier. A second rapid decay phase caused by the merger of the tertiary vortices is not observed due to the larger separation between primary and tertiary vortices. Note that for the late decay rates after $t^{*}=3$, the wind speed has only little impact, possibly because the limited size of the computational domain does not support the generation of large atmospheric eddies that may provide a lasting contribution to vortex decay. Further note, that the current simulations do not consider the onset of end-effects originating from touchdown. During final approach end effects may cause higher decay rates as they are observed here in this simplified computational setup [14]. The current LES agree well with the corresponding simulations of cases $\mathrm{N}$ and $\mathrm{H} 1$ in [31].
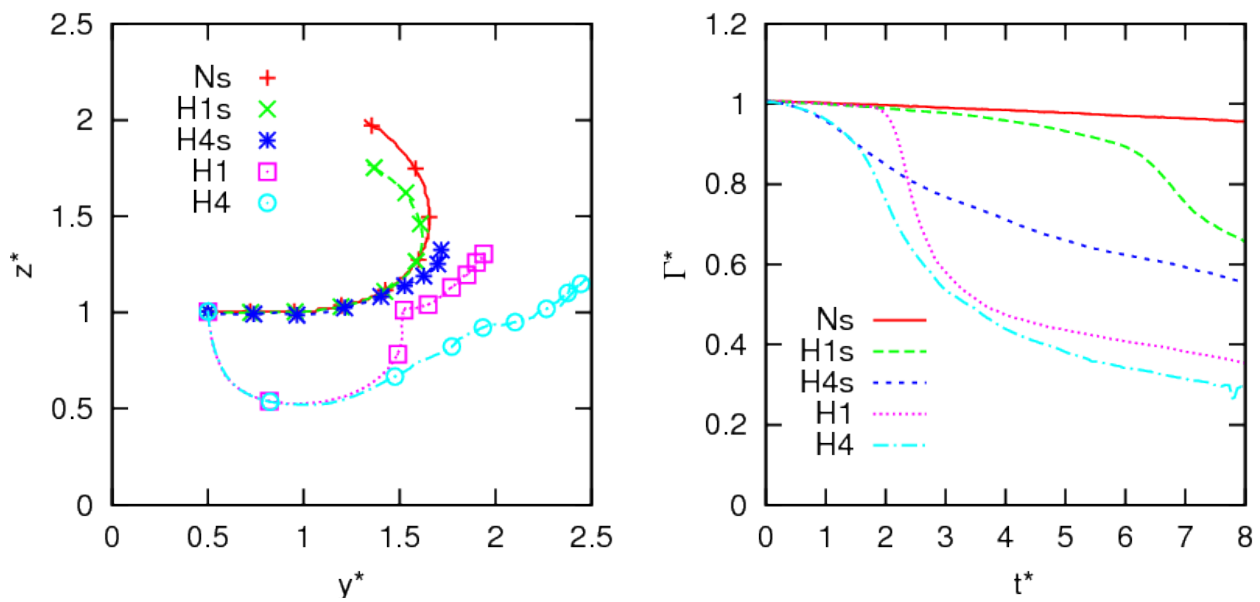

Fig. 4 Trajectories and circulation decay for single vortices and vortex pairs for different headwind strengths 


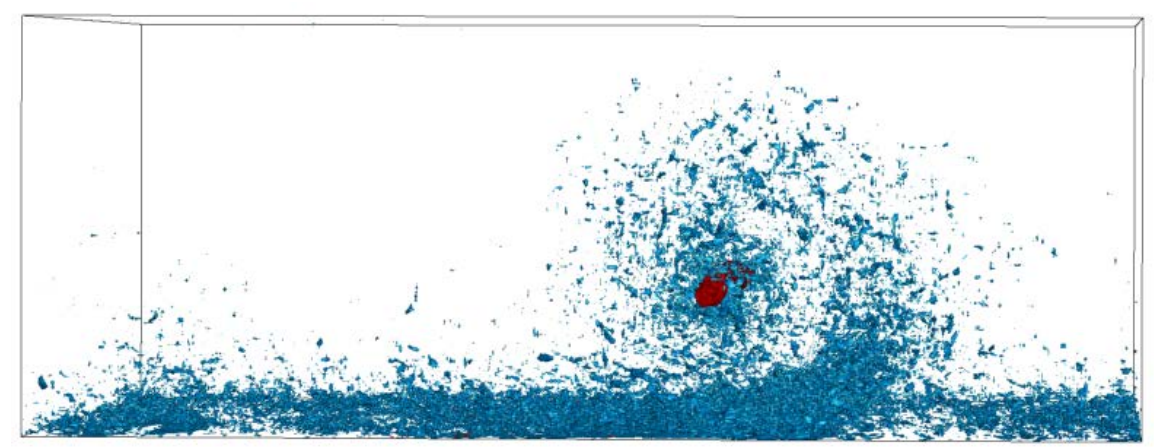

Fig. 5 Vorticity isosurfaces $\omega_{x}{ }^{*}=-31$ for single vortex in strong headwind at $t^{*}=4$ (case $\mathrm{H} 4 \mathrm{~s}$ )

Fig. 4 compares vortex trajectories and decay characteristics for single vortices and vortex pairs. Single vortices are included in the study in order to discriminate between effects caused by the mutual interaction between a vortex pair and the interaction of a single vortex with its environment. Therefore also the characteristic length scale $b_{0}$ taken from the vortex pair is retained for the single vortex. Due to the lack of their vortex mates, single vortices do not experience an initial downward motion but a pure lateral motion mutually induced by the single vortex and the vorticity layer generated at the ground. The respective transport speed is small because of the relatively large distance between the two vorticity agglomerations and almost independent from headwind speed and turbulence. It is noteworthy that even at the significant height of $b_{0}$ above ground a vorticity layer is generated at the ground that also detaches from the ground and starts to orbit around the primary vortex (see Fig. 5). Due to the large distance to the ground and thus the secondary vortex, this process is much slower than for vortex pairs approaching the ground actively and the rebound arc of the single vortices is much larger. Without wind the single vortex reaches a height of $2 b_{0}$ at a vortex age of $8 t_{0}$ without any substantial vortex decay. The computations are not continued beyond $t^{*}=$ 8. Note that during their substantial ascent the Ns and H1s vortices approach the free-slip boundary condition at $z^{*}=$ 3. This implies that the reverse lateral transport is somewhat overestimated, as they still possess a large amount of circulation. With increasing headwind the rebound speed and height decrease due to the loss of coherence of the secondary vortices caused by the disturbances of the environmental turbulence and the resulting vortex stretching effects exerted by the primary vortex on its planetary vortex [49].

In ground proximity rapid circulation decay sets in when the secondary vortex starts to merge with the primary vortex. Due to the smaller distance to the secondary vortices of vortex pairs this decay phase is reached much earlier by vortex pairs than by single vortices lingering at higher altitude. In contrast to the $1 w_{0}$ headwind cases the decay in the $4 w_{0}$ cases starts immediately and is identical for single vortices and vortex pairs. That is the initial decay is solely due to the erosion of the vortices by the environmental turbulence. Only at $t^{*}=1.7$ the vortex pair enters into the rapid decay phase related to the annihilation of primary and secondary vorticity. Later the decay rates of single vortices and vortex pairs again are similar and thus related to the interaction with environmental turbulence and the tertiary vortex. This is not surprising because during this decay regime the vortices both reside at similar altitudes close to $b_{0}$ and thus experience similar impacts of tertiary vortices and turbulent wind.

\section{Crosswind Effects on Single Vortices and Vortex Pairs}

In contrast to headwind crosswind causes asymmetric rebound characteristics. Firstly, the crosswind shear vorticity supports the formation of the secondary vorticity at the lee (downwind) vortex and attenuates it at the luff (upwind) vortex. Secondly, the primary vortices redistribute vorticity of the crosswind shear with which they mutually induce transport velocities depending on the ratios of the signs and the strengths of the primary and shear layer vorticity, respectively. These mechanisms are discussed and illustrated by numerical simulation results in this section in detail. The current LES agree well with the corresponding simulations of cases N, C1 and C2 in [31]. 

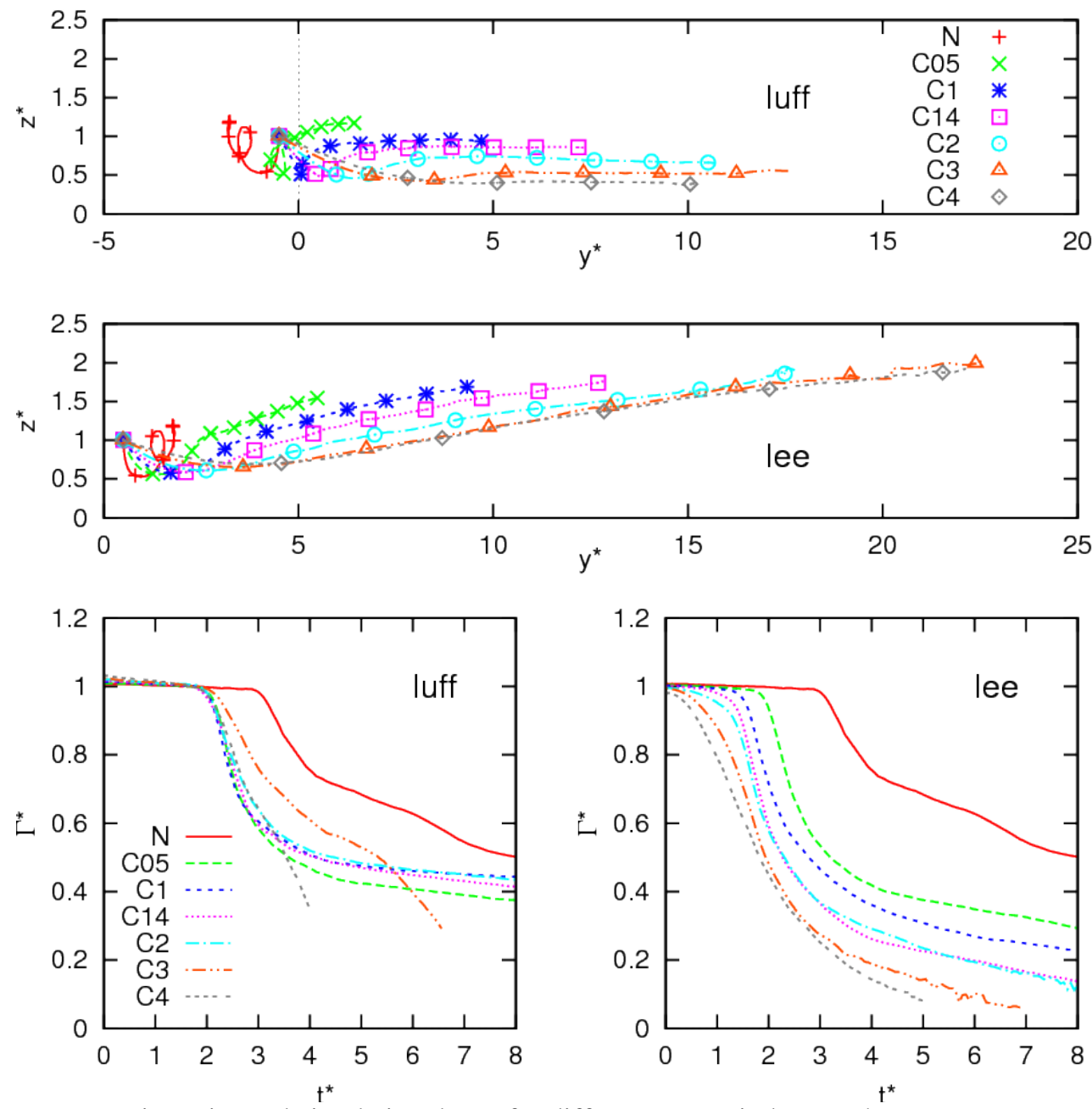

Fig. 6 Wake vortex trajectories and circulation decay for different crosswind strengths

Fig. 6 shows that the rebound characteristics strongly depend on the crosswind strength. The luff vortex rebound is highest at the weakest crosswind and decreases with increasing crosswind strength. In agreement with Doligalsky's considerations [11] the rebound is completely suppressed at a crosswind of $4 w_{0}$. For that case Doligalsky's parameter $\alpha$ (see below) achieves exactly its critical value of 0.75 . In contrast, the rebound height of the lee vortex increases continuously with increasing crosswind strength reaching a maximum height of $2 b_{0}$ in the 3 $w_{0}$ crosswind case. An even higher rebound height of the $4 w_{0}$ case is impeded by the accelerated decay.

A potentially most dangerous situation for following aircraft arises from wake vortices hovering above the runway because the self-induced lateral propagation velocity of the luff vortex is just compensated by the crosswind. According to Fig. 6 the luff vortex with a crosswind of $0.5 w_{0}$ roughly compensates the vortex-induced propagation speed and remains longest close to the runway centerline. This result is in perfect agreement with lidar measurements conducted at Frankfurt airport [3].

Note that the self-induced lateral speed of an inviscid point vortex at a height of $b_{0} / 2$ would be compensated exactly by a crosswind of $w_{0}$. The lower average lateral vortex speed of $0.5 w_{0}$ results from the early descent phase and the late rebound phase during which the vortex is higher than $b_{0} / 2$ and thus induces itself a lower lateral speed. The behaviour in ground proximity can be modelled employing point vortices by introducing first image vortices to mimic the divergence and then secondary vortices for the rebound $[3,25]$.

A wind of $0.5 w_{0}=0.77 \mathrm{~m} / \mathrm{s}=1.5$ knots corresponds to the Beaufort number of 1 "light air" where smoke drift indicates wind direction and leaves and wind vanes are stationary. In practice this potentially most dangerous situation for following aircraft may most likely be associated with still air conditions.

Rapid vortex decay of the luff vortex sets in independently from the crosswind strength at about $t^{*}=2$ (Fig. 6 left below). For crosswind speeds up to $2 w_{0}$ even the further circulation development is similar. In the cases C3 and C4 
the decay characteristics of the luff vortices deviate significantly from the lower wind speeds because the luff vortices linger so close to the ground that they link with it at $t^{*} \approx 6$ and 3.6, respectively. Ground linking may interfere with the interaction of secondary vorticity released from the ground and the primary vortices. Ground linking also makes tracking the vortex and, especially, computing its circulation more difficult as one cannot differentiate between circulation of the vortex itself and the same-signed vorticity of the wind shear. Hence, the circulation decay in cases C3 and C4 should be considered with some caution. However, the delayed decay brought along with ground linking is not operationally relevant because even the luff vortices are quickly advected away from the glide path at these crosswind speeds.

In contrast, the decay characteristics of the lee vortices clearly depend on the crosswind strength all over the considered velocity range. With increasing crosswind strength the onset of rapid decay is shifted from about $2 t_{0}$ at $0.5 w_{0}$ to earlier times and finally sets in without delay in the $4 w_{0}$ case. Also the decay rates after the rapid decay phase scale with the crosswind strength leading to quasi complete decay at $t^{*}=5$ for the strongest wind conditions. For the lee vortex the pronounced correlation of the onset time of rapid decay with the wind speed can be explained by the fact that both environmental turbulence and opposite signed wind shear vorticity are increasing with wind speed and accelerate vortex decay. For the luff vortex, however, the effect of increased turbulence is compensated by the increased same-signed wind shear vorticity strengthening the luff vortex with the result that its onset of rapid decay is independent from crosswind strength. Further, the turbulence generated by the interaction of the luff vortex with the ground may also further enhance the lee vortex decay.
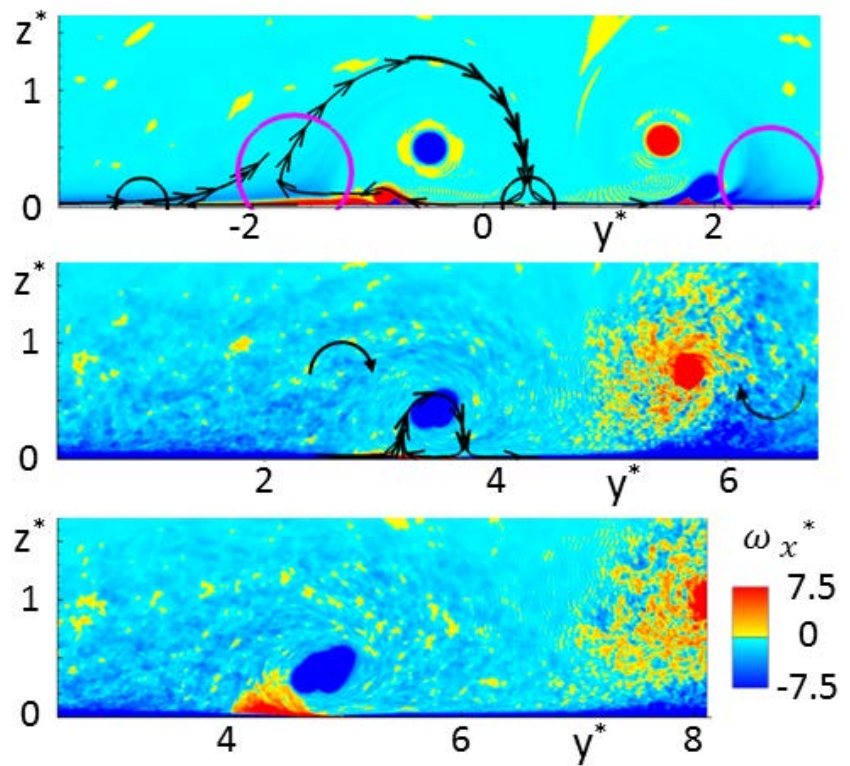

Fig. 7 Axial vorticity and streamlines of vortex pairs. Above: crosswind $0.5 w_{0}$ at $t^{*}=1.25$, crosswind $4 w_{0}$ at $t^{*}=$ 1.25 (center) and below at $t^{*}=1.8$

Fig. 7 delineates snapshots of axial vorticity and selected streamlines of vortex pairs that illustrate the mechanisms controlling vortex rebound. For the discussion of the rebound mechanisms we refer to the considerations introduced by Doligalsky [11] and Luton [13]. Doligalsky considers a single vortex advected in a uniform flow above a wall. Its fractional advection rate

$$
\alpha=\frac{V_{v}}{V_{0}}=1+\frac{\Gamma}{4 \pi z V_{0}}
$$

relates the velocity at which the vortex moves in the streamwise direction, $V_{v}$, to the cross-flow velocity $V_{0}$. Note that the circulation of the luff vortex attains negative circulation values. So for $\alpha=1$ the vortex is either very weak and/or far away from the surface and it is simply advected with the uniform flow. $\alpha=0$ corresponds to a vortex that is either sufficiently strong and/or close enough to the wall such that it remains stationary in the uniform flow.

For the luff vortex at a crosswind of $0.5 w_{0}$ and a vortex age of $t^{*}=1.25$ in Fig. 7 above, the corresponding $\alpha$ value is estimated to -1.2 . The negative sign of $\alpha$ indicates that the luff vortex propagates against the crosswind flow and exhibits an extended recirculation zone visualized by the black streamline. The significant length of the 
recirculation zone enables the generation of substantial counterrotating vorticity at the ground surface. Correspondingly, the detaching secondary vortex is quite strong and causes a significant rebound height (see Fig. 6).

At the strongest investigated crosswind of $4 w_{0}$ (see Fig. 7 center) $\alpha$ is estimated to 0.65 for the luff vortex at a vortex age of $t^{*}=1.8$; which is close to the critical value of $\alpha=0.75$, where the two stagnation points bounding the recirculation zone join to a single stagnation point situated at the surface. For $\alpha$-values above 0.75 no counterrotating vorticity is generated at the ground. The critical value of $\alpha=0.75$ is achieved precisely by the initial conditions of case C4 luff. In consistency with the diagnosed $\alpha$ value of 0.65 , the recirculation zone in Fig. 7 center is quite small. Due to the undiminished circulation and the low altitude above the surface, nevertheless, substantial secondary vorticity is generated at the ground as seen in Fig. 7 below. However, this positive vorticity does not detach from the wall, but is compensated by the approaching negative vorticity of the crosswind shear layer.

Luton [13] investigates the interaction of vortices with a boundary layer that are too weak to create vorticity at the wall. These weaker vortices, however, still redistribute the vorticity of the boundary layer, pulling it away from the surface. The redistributed vorticity alters the path of the primary vortex by mutual velocity induction. If the vorticity of the primary vortex and the crosswind vorticity have the same sign, which here is the case for the luff vortex, the vortex experiences a downward motion. Opposite signed vorticity results in an upward motion. We state that this effect always occurs within our investigated crosswind cases and depending on the wind conditions is superimposed to the effects of the generated secondary and tertiary vorticity.

There is evidence of this mechanism in all three parts of Fig. 7. At a crosswind of $0.5 w_{0}$ magenta circles mark the detaching boundary layer vorticity upwind of the luff vortex and downwind of the lee vortex. At $4 w_{0}$ the dark blue patchy (turbulent) detached boundary layer vorticity upwind and downwind of the vortex pair is clearly visible (rotational direction of vorticity indicated by black arrows). It is the interaction of the detached boundary layer vorticity with the primary vortex that enables the suppressed (continued) rebound of the luff (lee) vortex occurring at stronger crosswind speeds as displayed in Fig. 6.
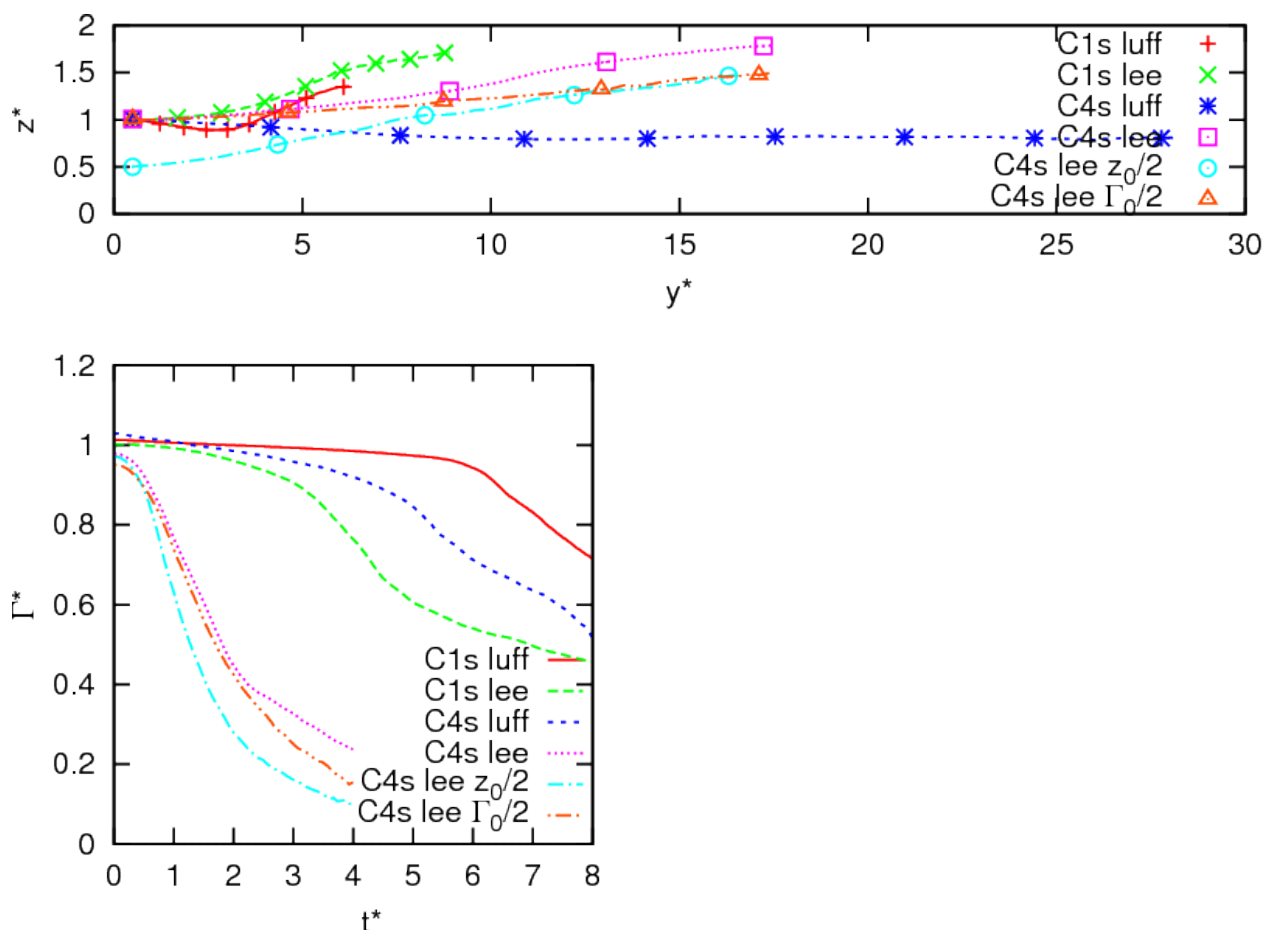

Fig. 8 Trajectories and circulation decay for single vortices at different crosswind strengths

Fig. 8 delineates vortex transport and decay of single vortices at crosswind strengths of $1 w_{0}$ and $4 w_{0}$ as well as different initial altitudes and initial circulation strengths. The notations luff and lee are used also for single vortices in order to denote their rotational directions with respect to the crosswind. Obviously all lee vortices ascend and all luff vortices descend at least initially as expected from Luton's considerations. 

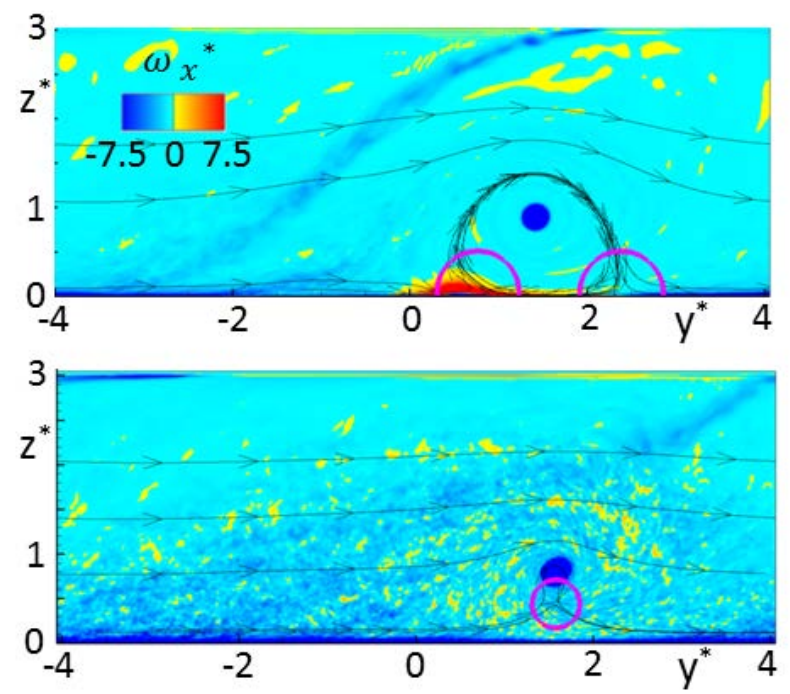

Fig. 9 Axial vorticity and streamlines of single luff vortices. Above: crosswind $w_{0}$ at $t^{*}=2.9$, below: crosswind $4 w_{0}$ at $t^{*}=0.6$

Doligalsky's $\alpha$-values of the luff vortices amount to 0.5 in case C1s and to 0.875 in case C4s. Fig. 9 corroborates that in the low crosswind case a recirculation zone exists which is connected with the ground surface and where a secondary vortex forms that later detaches and causes a rebound of the primary vortex. Accordingly, Fig. 8 above shows that the C1s luff vortex first descends until about $t^{*}=4$ driven by the redistribution of Luton's boundary layer vorticity and subsequently rebounds driven by the detached secondary vorticity predicted according to Doligalsky's considerations. The initial descent followed by a subsequent rebound corresponds to Luton's case III.

In case C4s, however, with the supercritical value of $\alpha=0.875$ the stagnation point of the recirculation zone is detached from the surface (Fig. 9 below) and thus the vortex conducts a continued descent driven by the redistribution of crosswind shear vorticity (Luton's case II).

The comparison of Fig. 6 and Fig. 8 indicates that the rebound of a single counterrotating vortex (see case C4s, lee, $z_{0} / 2$ ) is very similar to the lee vortex behaviour of a vortex pair (see case C4 lee). That is a large portion of the wake vortex behaviour in ground proximity is controlled by the interaction of the respective single vortex with its environmental flow which itself is characterized by the turbulence and shear that in turn is strongly impacted by the ground proximity. By implication its neighboring vortex is of minor importance once the vortex pair has separated sufficiently in ground effect.

All single lee vortices execute a continued ascent corresponding to Luton's case I. In case C1s lee the detachment of secondary vorticity supports the vortex ascent. Despite almost no detached secondary vorticity can be discriminated in the C4s lee case, its ascent is much faster, because there is more vorticity in the boundary layer that may lift the vortex. For all the $4 w_{0}$ lee vortex cases it is hard to discriminate the detachment of secondary vorticity from the turbulent boundary layer flow. But it is obvious that the redistributed boundary layer vorticity contributes dominantly to the ascent.

A comparison between the standard C4s lee vortex and the one initialized with halved circulation (C4s $\left.\Gamma_{0} / 2\right)$ shows that decreasing circulation decreases the ascent, as the induced velocity on the boundary layer is weaker and less secondary vorticity is lifted upwards. Lower initial altitude (C4s $\left.z_{0} / 2\right)$, however, increases the ascent, as the vortex is deeper within the shear layer which increases the intensity of the redistributed boundary layer vorticity.

The circulation evolution in Fig. 8 indicates that vortex decay is substantially faster for the lee vortex than the luff vortex. Obviously, the counterrotating boundary layer vorticity (lee vortex) is more efficient for vortex erosion than corotating vorticity (luff vortex). Further, the decay accelerates with crosswind strength and initial ground proximity. Notably, the decay rates correlate with the vertical propagation speeds. That is the intensity of the interaction of the primary vortices with the surrounding vorticity does not only cause different ascent or descent speeds but also determines the vortex decay rates. The circulation curves of the cases C4s lee with standard and halved initial circulation are almost identical as expected from the perspective of similarity theory for properly normalized quantities (cutting $\Gamma_{0}$ in half doubles $t_{0}$ ). 


\section{Effects of Diagonal Wind}

After the investigation of pure headwind and crosswind effects it is of interest to consider also the effects of arbitrary wind directions that is combinations of headwind and crosswind. For simplicity we consider diagonal wind, where headwind and crosswind have the same magnitude and analyze the resulting vortex behaviour. The modeling of wind effects in ground proximity for arbitrary wind directions is of vital interest for wake vortex advisory systems that optimize aircraft separations depending on the prevailing environmental conditions [50].

Fig. 10 above depicts vortex trajectories for different wind strengths and directions. The trajectories of the diagonal wind cases match very well with the crosswind cases where the crosswind strength corresponds to the crosswind component of the diagonal wind. Correspondingly, good agreement is found between the cases D14 and $\mathrm{C} 1$ as well as the cases D2 and C14. As mentioned in section 3 there is only a minor effect of headwind on vortex trajectories. Consequently, vortex trajectories in arbitrary wind situations can be modeled by simply considering the effect of the crosswind component.

In Fig. 10 below vortex decay characteristics of the three considered wind directions and wind speeds of $1.4 w_{0}$ and $2 w_{0}$ are shown. Good agreement with the decay rates of diagonal wind is achieved by averaging the decay rates of the corresponding headwind and crosswind cases with the same wind speed. This can be expressed by a simple formula according to $\Gamma\left(D_{w s, l u f f}\right)=\left[\Gamma\left(C_{w s, l u f f}\right)+\Gamma\left(H_{w s}\right)\right] / 2$ where the subscript ws denotes wind speed. Analogously this relation also holds for the lee vortex. Apparently, decay rates at arbitrary wind directions can be obtained by simple interpolation between the decay rates caused by the respective headwind and crosswind components.
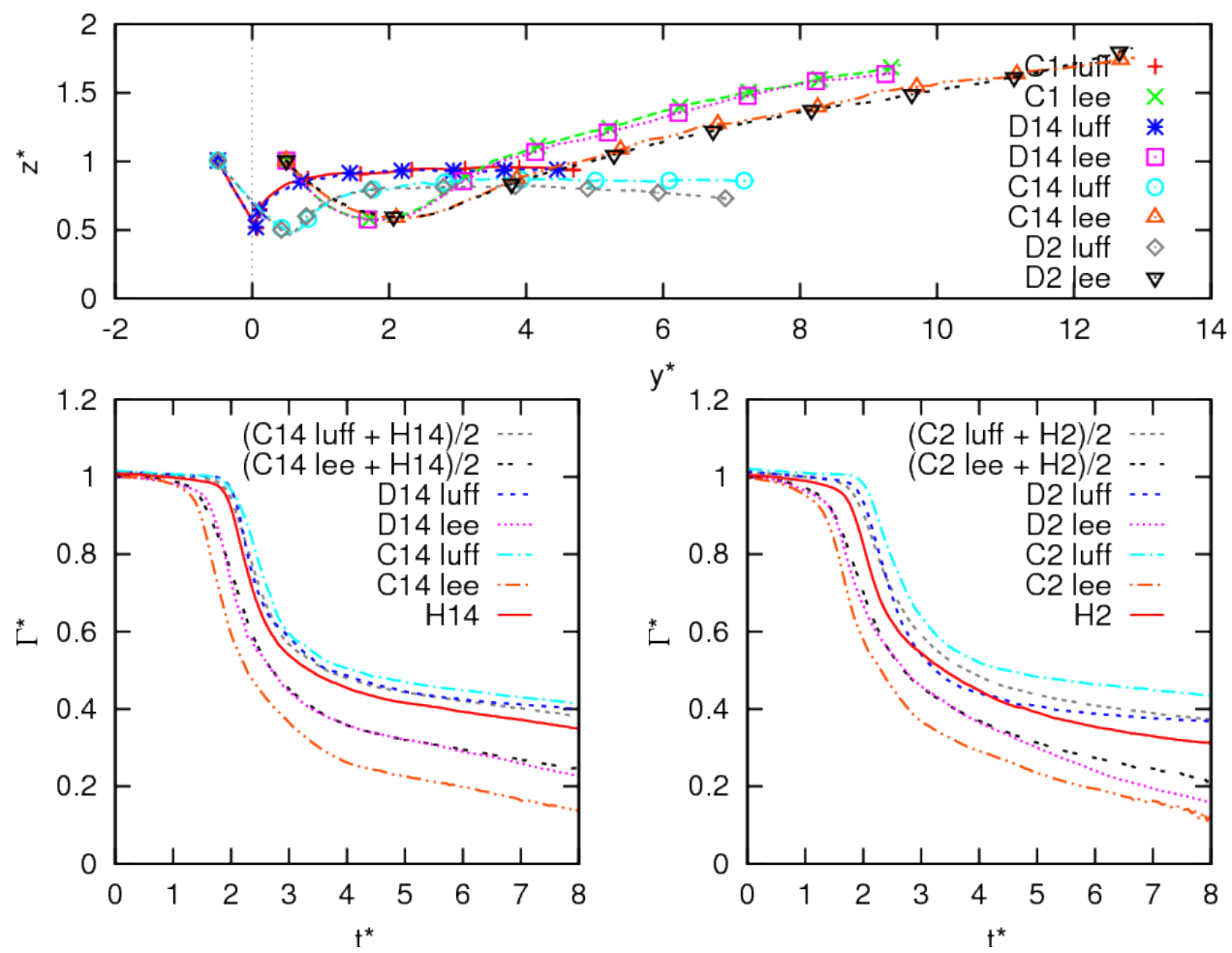

Fig. 10 Wake vortex trajectories and circulation decay for different wind directions

\section{Conclusion}

Wall-resolved large eddy simulations are employed to investigate the behaviour of counterrotating vortex pairs and single vortices in ground proximity at a variety of wind conditions. Single vortices are included in the study in order to discriminate between effects caused by the mutual interaction between a vortex pair and the interaction of a single vortex with its environment. Headwind, crosswind, and diagonal wind conditions at six different wind strengths are established by precomputations of turbulent half channel flows driven by prescribed pressure gradients. Then single vortices or counterrotating vortices are introduced by superimposing Lamb-Oseen vortices to the turbulent boundary layer flow. 
The impact of headwind is mainly restricted to the headwind turbulence that modifies vortex behaviour compared to the relatively simple vortex dynamics in a quiescent environment. With increasing headwind the enhanced turbulence increasingly disperses the coherence of the secondary vorticity formed at the ground such that the detachment of secondary vorticity becomes a more continuous process and the distinct rebound arcs observed in a less turbulent environment merge to a continuous rebound motion. Even at the significant height of one initial vortex pair separation single vortices still generate a vorticity layer at the ground that also detaches from the ground and starts to orbit around the primary vortex. Due to the large distance to the ground this process is much slower than for vortex pairs approaching the ground actively and the rebound arc of the single vortices is much larger.

For strong headwinds the decay rates prior to and after the rapid decay phase are very similar for single vortices and vortex pairs. During these two phases vortex decay is controlled mainly by the erosion of the vortices by the environmental turbulence. The intermediate rapid decay phase is related to the annihilation of primary and secondary vorticity and thus depends on the strength of the secondary vortices and, as a consequence, on the vortex height above ground.

In crosswind situations the rebound and decay characteristics strongly depend on the crosswind strength. A potentially most dangerous situation for following aircraft arises from wake vortices hovering above the runway because the self-induced lateral propagation velocity of the luff vortex is just compensated by the crosswind. In agreement with measurements conducted at Frankfurt airport, the current study identifies the most critical crosswind range around 0.5 initial vortex pair descent speeds.

In agreement with Doligalsky's considerations [11] the rebound of the luff vortex is completely suppressed at a crosswind of four initial vortex pair descent speeds. Beyond this crosswind level no recirculation zone forms along the ground in which secondary vortices could establish. In contrast, the rebound height of the lee vortex increases continuously with increasing crosswind strength reaching a maximum height that is only limited by vortex decay. Such a continued rebound cannot be explained by the formation of secondary vortices at the surface but by the concept of redistribution of boundary layer vorticity suggested by Luton [13]. The redistributed vorticity alters the path of the primary vortex by mutual velocity induction. If the vorticity of the primary vortex and the crosswind vorticity are of equal sign, the vortex experiences a downward motion. Opposite signed vorticity results in an upward motion. While this effect is predominant for single vortices it also occurs within our investigated vortex pair cases with crosswind. It is superimposed to the effects of the generated secondary and tertiary vorticity that forms along the ground depending on wind and vortex parameters.

For single vortices also the decay rates correlate with the vertical propagation speeds. The intensity of the interaction of the primary vortices with the surrounding vorticity does not only cause different ascent or descent speeds but also determines the vortex decay rates. We conclude that vortex ascent, descent, rebound and decay characteristics in a boundary layer are controlled by (i) the interaction of the vortices with secondary vorticity detaching from the ground, (ii) the redistribution of vorticity of the boundary layer which is altering the path of the primary vortices by mutual velocity induction, and (iii) the interaction of the vortices with the environmental turbulence.

Finally, the effects of combinations of headwind and crosswind on vortex behaviour are considered. Vortex trajectories in diagonal wind situations can be modeled simply by consideration of the respective crosswind component. Good agreement with the decay rates of diagonal wind is achieved by interpolation between the decay rates of the corresponding headwind and crosswind cases.

Acknowledgments We thank Prof. M. Manhart for the provision of the original version of the LES code MGLET. The provision of computation time on the supercomputer SuperMUC at the Leibniz-Rechenzentrum (LRZ) is gratefully acknowledged. The work was funded by the German Aerospace Research Center (DLR) projects Wetter \& Fliegen and L-bows.

\section{References}

1. Hallock, J. N., Greene, G. C., Burnham, D. C.: Wake Vortex Research - A Retrospective Look. Air Traffic Control Quart. 6, 161-178 (1998)

2. Eurocontrol: Challenges of Growth 2013. Summary Report, http://www.eurocontrol.int/statfor (2013). Accessed 6 July 2015

3. Holzäpfel, F., Steen, M.: Aircraft Wake-Vortex Evolution in Ground Proximity: Analysis and Parameterization, AIAA J. 45, 218-227 (2007) 
4. Dengler, K., Holzäpfel, F., Gerz, T., Wiegele, A., De Visscher, I., Winckelmans, G., Bricteux, L., Fischer, H., Konopka, J.: Crosswind Thresholds Supporting Wake-Vortex-Free Corridors for Departing Aircraft. Meteor. Appl. 19, 289-301 (2012). doi: 10.1002/met.261

5. Elsenaar B., van der Geest P., Speijker L., de Bruin A., Wolf S., Braun N., Gerz T., Holzäpfel F., Hahn K.-U., Schwarz C., Frech M., Köpp F., Mutuel L., Bourrez A., Barny H., Barbaresco F., Konopka J., Winckelmans G., Desenfans O., Pugh C., Davies H., Galpin D., Nicolaon J.-P., Vidal A., Harvey A., Wennerberg A., Schumacher J., Luckner R., Höhne G., Fuhrmann M., Laporte F., Hinsinger R., Schrauf G., Turp D., Agnew P., Hill C., Young R., Coustols E., Dolfi A., Jacquin L.: Wake Vortex Research Needs for "Improved Wake Vortex Separation Ruling" and "Reduced Wake Signatures", Part II, Specialists's Reports, Final Report of the Thematic Network "WakeNet2-Europe", 6th Framework Programme, 114 pages (2006)

6. Critchley, J., Foot, P.: UK CAA Wake Vortex Database: Analysis of Incidents Reported Between 1982 and 1990. Civil Aviation Authority, CAA Paper 91 (1991)

7. Rushton, D.: Preliminary results from FDR data collection campaign at London Heathrow, WakeNet-Europe 2015 Workshop, Amsterdam, URL: http://www.wakenet.eu/index.php?id=190 (2015). Accessed 6 July 2015

8. SAFO 14007: Safety Alert for Operators, Federal Aviation Administration, http://www.faa.gov/other_visit/aviation_industry/airline_operators/airline_safety/safo (2014). Accessed 6 July 2015

9. Treve, V.: EUROCONTROL Wake Program. WakeNet-Europe 2015 Workshop, Amsterdam, http://www.wakenet.eu/index.php?id=190 (2015). Accessed 6 July 2015

10. Holzäpfel, F., Dengler, K., Gerz, T. Schwarz, C.: Prediction of Dynamic Pairwise Wake Vortex Separations for Approach and Landing. AIAA Paper 2011-3037 (2011)

11. Doligalski, T. L., Smith, C. R., Walker, J. D. A.: Vortex Interactions with Walls. Annu. Rev. Fluid Mech. 26, 573-616 (1994)

12. Stephan, A., Holzäpfel, F., Misaka, T.: Aircraft Wake-Vortex Decay in Ground Proximity - Physical Mechanisms and Artificial Enhancement. J. Aircraft 50, 1250-1260 (2013). doi/abs/10.2514/1.C032179

13. Luton, A., Ragab, S., Telionis, D.: Interaction of spanwise vortices with a boundary layer,” Phys. Fluids 7, 2757-2765 (1995)

14. Stephan, A., Holzäpfel, F., Misaka, T.: Hybrid simulation of wake-vortex evolution during landing on flat terrain and with plate line. Int. J. Heat Fluid Flow 49, 18-27 (2014). doi:10.1016/j.ijheatfluidflow.2014.05.004

15. Prandtl, L.: Tragflächen-Auftrieb und -Widerstand in der Theorie. Ergebnisse der Aerodynamischen Versuchsanstalt zu Göttingen, I. Lieferung, 35 - 39 (1921)

16. Wieselsberger, C., 1921. Über den Flügelwiderstand in der Nähe des Bodens. Zeitschrift für Flugtechnik und Motorluftschiffahrt 10, 145-147 (1921)

17. Dee, F.S., Nicholas, O.P.: Flight Measurement of Wing Tip Vortex Motion near the Ground. CP 1065, British Aeronautical Research Council (1968)

18. Harvey, J. K., Perry, F. J.: Flowfield Produced by Trailing Vortices in the Vicinity of the Ground. AIAA J. 9, 1659-1660 (1971)

19. Barker, S. J., Crow, S. C.: The Motion of Two-Dimensonal Vortex Pairs in a Ground Effect. J. Fluid Mech. 82, 659-671 (1977)

20. Orlandi, P.: Vortex Dipole Rebound from a Wall. Phys. Fluids A 2, 1429-1436 (1990)

21. Schilling, V. K.: Motion and Decay of Trailing Vortices Within the Atmospheric Surface Layer. Beitr. Phy. Atmos. 65, 157-169 (1992)

22. Robins, R. E., Delisi, D. P.: Potential Hazard of Aircraft Wake Vortices in Ground Effect with Crosswind. J. Aircraft 30, 201-206 (1993)

23. Zheng, Z. C., Ash, R. L.: Study of Aircraft Wake Vortex Behavior Near the Ground. AIAA J. 34, $580-589$ (1996)

24. Corjon, A., Poinsot, T.: Behavior of Wake Vortices Near Ground. AIAA J. 35, 849-855 (1997).

25. De Visscher, I., Lonfils, T., Winckelmans, G.: Fast-Time Modeling of Ground Effects on Wake Vortex Transport and Decay. J. Aircraft 50, 1514-1525 (2013)

26. Corjon, A., Stoessel, A.: Three-Dimensional Instability of Wake Vortices Near the Ground. AIAA Paper 971782 (1997)

27. Luton, J. A., Ragab, S. A.: The Three-Dimensional Interaction of a Vortex Pair with a Wall. Phys. Fluids 9, 2967-2980 (1997)

28. Proctor, F. H., Hamilton, D. W., Han, J.: Wake Vortex Transport and Decay in Ground Effect: Vortex Linking with the Ground. AIAA Paper 2000-0757 (2000)

29. Hamilton, D. W., Proctor, F. H.: Wake Vortex Transport in the Proximity of the Ground. Proc. 19th Digital Avionics Conf., AIAA and IEEE, Philadelphia, PA, 1-8 (2000)

30. Spalart, P. R., Strelets, M. Kh., Travin, A. K., Shur, M. L.: Modeling the Interaction of a Vortex Pair with the Ground. Fluid Dyn. 36, 899-908 (2001)

31. Giovannini, A., Georges, L., Geuzaine, P., Duponcheel, M., Bricteux, L., Lonfils, T., Winckelmans, G.: Effect of wind conditions on the evolution of a two-vortex system near the ground. Fundamental Research on Aircraft Wake Phenomena, Rept. AST4-CT-2005-012238 (2007). http://far-wake.irphe.univmrs.fr/IMG/deliverables/deliverables.html 
32. Liu, H.-T., Hwang, P. A., Srnsky, R. A.: Physical Modeling of Ground Effects on Vortex Wakes. J. Aircraft 29, 1027-1034 (1992)

33. Köpp, F.: Doppler Lidar Investigation of Wake Vortex Transport Between Closely Spaced Parallel Runways. AIAA J. 32, 805-810 (1994)

34. Hallock, J. N., Osgood, S. P., Konopka, J.: Wake Vortex Effects on Parallel Runway Operations. AIAA Paper 2003-0379 (2003)

35. Burnham, D. C., Hallock, J. N.: Measurements of Wake Vortices Interacting with the Ground. J. Aircraft 42 1179-1187 (2005)

36. Holzäpfel, F., Stephan, A., Heel, T., Körner, S.: Enhanced Wake Vortex Decay in Ground Proximity Triggered by Plate Lines. Aircraft Eng. Aeros. Techn. (2015). doi: 10.1108/AEAT-02-2015-0045

37. Holzäpfel, F., Tchipev, N., Stephan, A.: Wind Impact on Single Vortices and Counterrotating Vortex Pairs in Ground Proximity. AIÁA Paper 2015-3174 (2015)

38. Tchipev, N.: Numerical Simulation of Wind Impact on Transport and Decay of Wake Vortices of Landing Aircraft,” Master's Thesis, Computational Science and Engineering, Technische Universität München, Munich, Germany (2013)

39. Manhart, M.: A Zonal Grid Algorithm for DNS of Turbulent Boundary Layer. Computer \& Fluids 33, 435-461 (2004)

40. Hokpunna A., Manhart, M.: Compact Fourth-order Finite Volume Method for Numerical Solutions of NavierStokes Equations on Staggered Grids. J. Comput. Phys. 229, 7545-7570 (2010)

41. Hirt, C. W., Cook, J. L.: Calculating Three-dimensional Flows Around Structures and Over Rough Terrain. J. Comput. Phys. 10, 324-340 (1972)

42. Williamson, J. H.: Low-Storage Runge-Kutta Schemes. J. Comput. Phys. 35, $48-56$ (1980)

43. Meneveau, C., Lund, T. S., Cabot, W. H.: A Lagrangian Dynamic Subgrid-scale Model of Turbulence. J. Fluid Mech. 319, 353-385 (1996)

44. Gerz T., Holzäpfel F., Darracq D.: Commercial Aircraft Wake Vortices. Prog. Aerosp. Sci. 38, 181-208 (2002)

45. Misaka, T., Obayashi, S., Stephan, A., Holzäpfel, F., Gerz, T., Nakahashi, K.: Numerical Simulation of JetWake Vortex Interaction. AIAA Paper 2014-0926 (2014)

46. de Bruin, A., Winckelmans, G.: Cross-flow kinetic energy and core size growth of analytically defined wake vortex pairs. Technical Report, NLR-CR-2005-412, NLR (2005)

47. Delisi, D. P., Greene, G.C., Robins, R.E., Vicroy, D.C., Wang, F.Y.: Aircraft Wake Vortex Core Size Measurements. AIAA Paper 2003-3811 (2003)

48. Holzäpfel, F., Gerz, T., Köpp, F., Stumpf, E., Harris, M., Young, R. I., Dolfi-Bouteyre, A.: Strategies for Circulation Evaluation of Aircraft Wake Vortices Measured by Lidar. J. Atmos. Oceanic Technol. 20, 11831195 (2003)

49. Holzäpfel, F., Stephan, A., Körner, S., Misaka, T.: Wake Vortex Evolution During Approach and Landing With and Without Plate Lines. AIAA Paper 2014-0925 (2014)

50. Holzäpfel, F., Gerz, T., Frech, M., Tafferner, A., Köpp, F., Smalikho, I., Rahm, S., Hahn, K.-U., Schwarz, C.: The Wake Vortex Prediction and Monitoring System WSVBS - Part I: Design. Air Traffic Control Quart. 17, 301-322 (2009) 